VOL. 29 (1984), 303-308.

\title{
ALGEBRAS GENERATED BY \\ SYMMETRIC IDEMPOTENTS
}

\author{
David Choate
}

\begin{abstract}
Let $F$ be a field. If $A$ is an $F$-algebra with involution that is generated (as a space) by symmetric idempotents, then $A$ is a subdirect product of copies of $F$ if and only if every idempotent in $A$ is symmetric.
\end{abstract}

\section{Introduction}

This paper arose from the study of the questions raised by Herstein [2] concerning when the vector space generated by the symetric idempotents in a simple ring with involution is equal to itself. If $S$ is a simple ring and $C(S)$ the centroid of $S$, then $C(S)$ is a field and $S$ is a $C(S)$-algebra. Let $E^{*}(S)$ be the $C(S)$-subspace generated by the non-zero symmetric idempotents. Chaung and Lee [1, Example 4$]$ showed that $E^{*}(S)$ can be a ring and yet not be $S$ itself. Observe that if $E^{*}(S)$ is a ring, then $E^{*}(S)$ is an algebra generated as a vector space by symmetric idempotents, the object of our investigation.

Let $F$ be a field. In this paper we show that if $A$ is an $F-$. algebra with involution * that is generated (as a space) by symetric idempotents, then $A$ is a subdirect product of copies of $F$ if and only if every idempotent in $A$ is symmetric.

Received 14 November 1983.

Copyright Clearance Centre, Inc. Serial-fee code: 0004-9727/84 $\$ \mathrm{~A} 2.00+0.00$. 


\section{A commutivity condition}

In this section we require only that $A$ be an $F$-algebra generated by idempotents. If we ask when $A$ is commutative, then we are led to

THEOREM 1. Suppose $F$ is a field and $A$ is an F-algebra generated by idempotents. The folzowing are equivalent:

(i) A is commutative;

(ii) $A$ has no non-zero nilpotent elements;

(iii) $A$ is F-isomorphic to a subdirect product of copies of $F$.

Proof. Take $A$ to be commutative. We let $I$ denote the set of nonzero idempotents in $A$. Any non-zero element in $A$ can be written in the form $\lambda_{1} e_{1}+\ldots+\lambda_{n} e_{n}$ where $0 \neq \lambda_{i} \in F, 0 \neq e_{i} \in I, e_{i}^{\prime s}$ distinct, and $n$ is minimal. We call $n$ the length of the element. If $A$ has a non-zero nilpotent element, then choose one of minimal length among all such elements. Denote the element by $w$ and express it as above. So for each $i=1, \ldots, n$,

$$
w-w e_{i}=\sum \lambda_{j}\left(e_{j}^{\left.-e_{j} e_{i}\right)},\right.
$$

where $j=1, \ldots, n$ and $j \neq i$, is an element of length less than $n$ or $w-w e_{i}$ is zero. But $w-w e_{i}$ is nilpotent; so the latter must hold and $w=w e_{i} \cdot$ Observe that

$$
w^{2}=\left(\lambda_{1}+\ldots+\lambda_{n}\right) w
$$

and then inductively we have

$$
0=w^{k}=\left(\lambda_{1}+\ldots+\lambda_{n}\right)^{k-1} w,
$$

where $k$ is the index of nilpotency of $w$. Consequently,

$$
\left(\lambda_{1}+\ldots+\lambda_{n}\right)^{k-1}=0
$$

or

$$
\lambda_{1}+\ldots+\lambda_{n}=0
$$


Let $x_{j}$ be the product of the idempotents $e_{j}, e_{j+1}, \ldots, e_{n}$, $j=1, \ldots, n$. Then

$$
w x_{1}=w e_{1} x_{2}=w x_{2}=\ldots=w e_{n}=w,
$$

but

$$
\begin{aligned}
w x_{1} & =\left(\lambda_{1} e_{1}+\ldots+\lambda_{n} e_{n}\right) x_{1} \\
& =\lambda_{1} x_{1}+\ldots+\lambda_{n} x_{1} \\
& =\left(\lambda_{1}+\ldots+\lambda_{n}\right) x_{1} \\
& =0 .
\end{aligned}
$$

So $w=0$ and $A$ has no non-zero nilpotent elements. Thus (i) implies (ii).

One obtains ( $i$ ii) from (ii) by recalling that in a ring without nilpotent elements the idempotents are central. So we may consider $A$ to be commutative and without nilpotent elements. Using an F-algebra version of the Krull-McCoy Theorem, that a ring without nilpotent elements is isomorphic to a subdirect product of integral domains, we have that $A$ is a subdirect product of $F$-algebras, $A_{i}, i$ running over some index set $\Lambda$, where each $A_{i}$ is without zero divisors. Each $A_{i}$, being an $F_{-}$ homomorphic image of $A$, must also be generated as an $F$-vector space by idempotents. Since $A_{i} \neq(0)$, it contains a non-zero idempotent. But since $A_{i}$ is a ring without zero divisors, this idempotent is a unit element, say $1_{i}$. In fact, since the idempotents in $A$ must go into 0 or $1_{i}$ under the $i$ th projection $F$-homomorphism, each element of $A_{i}$ is of the form $I_{i} \cdot \lambda \in F$ and consequently $A_{i}$ is a field which is isomorphic to $F$.

It is immediate that (iii) implies (i).

A corollary to this theorem is of interest when $A$ is noncommutative.

COROLLARY 1. Suppose $F$ be a field and $A$ is an F-algebra generated by idempotents. All of the nilpotent elements in $A$ are formd in its commutator ideal.

Proof. Let $C$ be the commutator ideal of $A$. Then $A / C$ is a 
commutative $F$-algebra generated by idempotents. If $n$ is a nilpotent element in $A$, then $n+C$ is a nilpotent element in $A / C$. By Theorem 1 we must have $n+C=C$, or $n \in C$.

\section{A *-version}

We now suppose that $A$ is an $F$-algebra with involution * that is generated by symmetric idempotents and ask when $A$ is commutative.

THEOREM 2. Suppose $F$ is a field and $A$ is an F-algebra with involution generated by symmetric idempotents. The algebra $A$ is commutative if and only if every idempotent in $A$ is symmetric.

Proof. Suppose every idempotent in $A$ is symmetric. Then if $e_{1}$ and $e_{2}$ are idempotents in $A$, so is $e_{1}+e_{1} e_{2}-e_{1} e_{2} e_{1}$. Then we must have

$$
\begin{aligned}
& e_{1}+e_{1} e_{2}-e_{1} e_{2} e_{1}=\left(e_{1}+e_{1} e_{2}-e_{1} e_{2} e_{1}\right) * \\
& =e_{1}^{*}+e_{2}^{*} e_{1}^{*}-e_{1}^{*} e_{2}^{*} e_{1}^{*} \\
& =e_{1}+e_{2} e_{1}-e_{1} e_{2} e_{1} \text {. }
\end{aligned}
$$

Consequently, $e_{1} e_{2}=e_{2} e_{1}$ for any two symmetric idempotents in $A$. This is enough to show that $A$ is commutative.

Now suppose that $A$ is commutative. We let $S$ denote the set of non-zero symmetric idempotents in $A$. Any non-zero element in $A$ can be written in the form $\lambda_{1} e_{1}+\ldots+\lambda_{n} e_{n}$ where $0 \neq \lambda_{i} \in F, 0 \neq e_{i} \in S$, $e_{i}$ 's distinct, $n$ minimal. We call $n$ the length of the element. If $A$ has an idempotent that is not symmetric, then choose one of minimal length among all such elements. Denote this element by $e$ and express it as above. So for each $i=1, \ldots, n$,

$$
e-e e_{i}=\sum \lambda_{j}\left(e_{j}-e_{j} e_{i}\right) \text {, }
$$

where $j=1, \ldots, n$ and $j \neq i$, is an idempotent of length less than $n$, and hence $e-e e_{i}$ must be symmetric. So we know

$$
e-e e_{i}=e^{*}-e^{*} e_{i}
$$


for each $i$. Multiplying by $\lambda_{i}$ we have

$$
\lambda_{i} e-e\left(\lambda_{i} e_{i}\right)=\lambda_{i} e^{*}-e^{*}\left(\lambda_{i} e_{i}\right)
$$

Summing over $i$ from 1 to $n$ we get

$$
\left(\lambda_{1}+\ldots+\lambda_{n}\right) e-e=\left(\lambda_{1}+\ldots+\lambda_{n}\right) e^{*}-e^{*} e .
$$

If $\lambda_{1}+\ldots+\lambda_{n}=0$ then $e=e^{*} e$ which implies $e$ is symmetric. If $\lambda_{1}+\ldots+\lambda_{n}=1$, then $e^{*} e=e^{*}$. So $e=e^{*} e$. Thus we may assume below that

$$
\lambda_{1}+\ldots+\lambda_{n} \neq 0,1
$$

Let $x_{i}$ be the product of the idempotents $e_{i}, e_{i+1}, \ldots, e_{n}$, $i=1, \ldots, n$. If we multiply

$$
e=\lambda_{1} e_{1}+\ldots+\lambda_{n} e_{n}
$$

by $x_{1}$, then we obtain

$$
\begin{aligned}
e x_{1} & =\lambda_{1} x_{1}+\ldots+\lambda_{n} x_{1} \\
& =\left(\lambda_{1}+\ldots+\lambda_{n}\right) x_{1} .
\end{aligned}
$$

After squaring and subtracting we have

$$
\left[\left(\lambda_{1}+\ldots+\lambda_{n}\right)^{2}-\left(\lambda_{1}+\ldots+\lambda_{n}\right)\right] x_{1}=0 \text {. }
$$

This implies $x_{1}=0$. Now set $e-e e_{i}=s_{i}, i=1, \ldots, n$. Then

$$
\begin{aligned}
0 & =e x_{1} \\
& =e e_{1} x_{2} \\
& =e-s_{1} x_{2} \\
& =e x_{2}-s_{1} x_{2} \\
& =e e_{2} x_{3}-s_{1} x_{2} \\
& =e x_{3}-s_{2} x_{3}-s_{1} x_{2} \\
& \vdots \\
& =e-s_{n}-s_{n-1} x_{n}-s_{n-2} x_{n-1}-\ldots-s_{1} x_{2} .
\end{aligned}
$$


Since $s_{i}$ and $x_{i}, i=1, \ldots, n$, are symetric elements, $e$ is symetric.

COROLLARY 2. Suppose $F$ is a field and $A$ is an $F$-algebra with involution * generated by symmetric idempotents. If $e$ is an idempotent in $A$, then $e-e^{*}$ is an element in its commutator ideal.

Proof. We again denote the commutator ideal of $A$ by $C$. Define $C^{*}=\left\{c^{*} ; c \in C\right\}$. Since $C=C^{*}$ we know $A / C$ is a commutative $F$ algebra having involution which is generated by symetric idempotents. If $e$ is an idempotent in $A$, then $e+C$ is an idempotent in $A / C$. By Theorem 2 we know $e+C=e^{*}+C$, or $e-e^{*} \in C$.

If we combine Theorem $I$ and Theorem 2, then we immediately obtain

THEOREM 3. Suppose $F$ is a field and $A$ is an F-algebra with involution generated by symmetric idempotents. The following are equivalent:

(i) $A$ is commutative;

(ii) every idempotent in $A$ is symmetric;

(iii) $A$ has no non-zero nilpotent elements;

(iv) $A$ is F-isomorphic to a subdirect product of copies of $F$.

\section{References}

[1] C.L. Chaung and P.H. Lee, "Idempotents in simple rings", J. Algebra 56 (1979), 510-515.

[2] I.N. Herstein, Rings with involution (University of Chicago Press, Chicago, 1979).

Department of Mathematics,

Xavier University of Louisiana,

New Orleans,

Louisiana 70125 ,

USA. 